\title{
On Local Times: Application to Pricing Using Bid-Ask
}

\author{
Paul C. Kettler ${ }^{1}$, Olivier Menoukeu-Pamen ${ }^{2}$, Frank Proske ${ }^{1}$ \\ ${ }^{1}$ Centre of Mathematics for Applications, Department of Mathematics, University of Oslo, Oslo, Norway \\ ${ }^{2}$ Institute for Financial and Actuarial Mathematics, Department of Mathematics, University of Liverpool, Liverpool, UK \\ Email: paulck@math.uio.no, Menoukeu@liverpool.ac.uk, proske@math.uio.no
}

Received October 12, 2013; revised December 3, 2013; accepted January 4, 2014

Copyright (c) 2014 Paul C. Kettler et al. This is an open access article distributed under the Creative Commons Attribution License, which permits unrestricted use, distribution, and reproduction in any medium, provided the original work is properly cited. In accordance of the Creative Commons Attribution License all Copyrights (C) 2014 are reserved for SCIRP and the owner of the intellectual property Paul C. Kettler et al. All Copyright @ 2014 are guarded by law and by SCIRP as a guardian.

\begin{abstract}
In this paper, we derive the evolution of a stock price from the dynamics of the "best bid" and "best ask". Under the assumption that the bid and ask prices are described by semimartingales, we study the completeness and the possibility for arbitrage on such a market. Further, we discuss (insider) hedging for contingent claims with respect to the stock price process.
\end{abstract}

\section{KEYWORDS}

\section{Order Statistics; Semimartingales; Local Times; Arbitrage}

\section{Introduction}

The theory of asset pricing and its fundamental theorem were initiated in the Arrow-Debreu model, the Black and Scholes formula, and the Cox and Ross model. They have now been formalized in a general framework by Harrison and Kreps [1], Harrison and Pliska [2], and Kreps [3] according to the no arbitrage principle. In the classical setting, the market is assumed to be frictionless i.e. a no arbitrage dynamic price process is a martingale under a probability measure equivalent to the reference probability measure.

However, real financial markets are not frictionless, and so an important literature on pricing under transaction costs and liquidity risk has appeared. See [4,5] and references therein. In these papers the bid-ask spreads are explained by transaction costs. Jouini and Kallal in [5] in an axiomatic approach in continuous time assigned to financial assets a dynamic ask price process (respectively, a dynamic bid price process). They proved that the absence of arbitrage opportunities is equivalent to the existence of a frictionless arbitrage-free process lying between the bid and the ask processes, i.e., a process which could be transformed into a martingale under a well-chosen probability measure. The bid-ask spread in this setting can be interpreted as transaction costs or as the result of entering buy and sell orders.

Taking into account both transaction costs and liquidity risk, Bion-Nadal in [4] changed the assumption of sublinearity of ask price (respectively, superlinearity of bid price) made in [5] to that of convexity (respectively, concavity) of the ask (respectively, bid) price. This assumption combined with the time-consistency property for dynamic prices allowed her to generalize the result of Jouini and Kallal [5]. She proved that the "no free lunch" condition for a time-consistent dynamic pricing procedure [TCPP] is equivalent to the existence of an equivalent probability measure $Q$ that transforms a process between the bid and ask processes of any financial instrument into a martingale. See also Cherny [6] regarding the characterization of non-existence of arbitrage opportunities for stock prices constructed from bid and ask processes.

In recent years, a pricing theory has also appeared taking inspiration from the theory of risk measures. First to investigate in a static setting were Carr, Geman, and Madan [7] and Föllmer and Schied [8]. The point of view of pricing via risk measures was also considered in a dynamic way using backward stochastic differential equations [BSDE] by El Karoui and Quenez [9], El Karoui, Peng, and Quenez [10], and Peng [11,12]. This theory 
soon became a useful tool for formulating many problems in mathematical finance, in particular for the study of pricing and hedging contingent claims [10]. Moreover, the BSDE point of view gave a simple formulation of more general recursive utilities and their properties, as initiated by Duffie and Epstein (1992) in their [stochastic differential] formulation of recursive utility [10].

In the past, in real financial markets, the load of providing liquidity was given to market makers, specialists, and brokers, who trade only when they expect to make profits. Such profits are the price that investors and other traders pay, in order to execute their orders when they want to trade. To ensure steady trading, the market makers sell to buyers and buy from sellers, and get compensated by the so-called bid-ask spread. The most common price for referencing stocks is the last trade price. At any given moment, in a sufficiently liquid market there is a best or highest "bid" price, from someone who wants to buy the stock and there is a best or lowest "ask" price, from someone who wants to sell the stock. The best bid price $R(t)$ and best ask (or best offer) price $T(t)$ are the highest buying price and the lowest selling price at any time $t$ of trading.

In the present work, we consider models of financial markets in which all parties involved (buyers, sellers) find incentives to participate. Our framework is different from the existing approach (see [4,5] and references therein) where the authors assume some properties (sublinearity, convexity, etc.) on the ask (respectively, bid) price function in order to define a dynamic ask (respectively, bid). Rather, we assume that the different bid and ask prices are given. Then the question we address is how to model the "best bid" (respectively, the "best ask") price process with the intention to obtain the stock price dynamics.

The assumption that the bid and ask processes are described by (continuous) semimartingales in our special setting entails that the stock price admits arbitrage opportunities. Further, it turns out that the price process possesses the Markov property, if the bid and ask are Brownian motion or Ornstein-Uhlenbeck type, or more generally Feller processes. Note that our results are obtained without assuming arbitrage opportunities.

This paper is also related with [13] where the authors explore market situations where a large trader causes the existence of arbitrage opportunities for small traders in complete markets. The arbitrage opportunities considered are "hidden" which means that they are almost not observable to the small traders, or to scientists studying markets because they occur on time sets of Lebesgue measure zero.

The paper is organized as follows: Section 2 presents the model. Section 3 studies the Markovian property of the processes, while Sections 4 and 5 are devoted to the study of completeness, arbitrage and (insider) hedging on a market driven by such processes.

\section{The Model}

Let $B(s)=\left(B(s)^{1}, \cdots, B(s)^{n}\right)^{\mathrm{T}} \quad$ (where $(\cdot)^{\mathrm{T}}$ denotes transpose) be a $n$-dimensional standard Brownian motion on a filtered probability space $\left(\Omega, \mathscr{T},\left\{\mathscr{F}_{t}\right\}_{0 \leq t \leq T}, P\right)$.

Suppose bid and ask price processes $\left\{X_{i}(t)\right\}_{0 \leq t \leq T}, 1 \leq i \leq n$, which are modeled by continuous semimartingales

$$
X_{i}(t)=X_{i}(0)+\int_{0}^{t} a_{i}\left(s, X_{s}, \omega\right) \mathrm{d} s+\int_{0}^{t} \sigma\left(s, X_{s}, \omega\right) \mathrm{d} B_{i}(s) .
$$

Here we consider the following model for bid and ask prices. See Figure 1.

The evolution of the stock price process $S(t)$ is based on $X_{i}(t), i=1, \cdots, n$. Denote by $\operatorname{Bid}(t)$ the Best Bid and $A s k(t)$ the Best Ask at time $t$. Then $\operatorname{Bid}(t)$ is the lowest price that a day trader seller is willing to accept for a stock at that time and $A s k(t)$ is the highest price that a day trader buyer is willing to pay for that stock at any particular point in time. Let us define the processes $X^{+}(t)=\max (X(t), 0)$ and $X^{*}(t)=\min (X(t), 0)$. Further set

$$
\begin{aligned}
R(t) & :=\min _{1 \leq i \leq n} X_{i}^{+}(t), \\
T(t) & :=\max _{1 \leq i \leq n} X_{i}^{*}(t) .
\end{aligned}
$$

where we use the convention that $\min \{\varnothing\}=0$ and $\max \{\varnothing\}=0$. Then $\operatorname{Bid}(t)$ and $\operatorname{Ask}(t)$ can be modeled as

$$
\operatorname{Bid}(t):=\min \{R(t),-T(t)\}
$$

and 


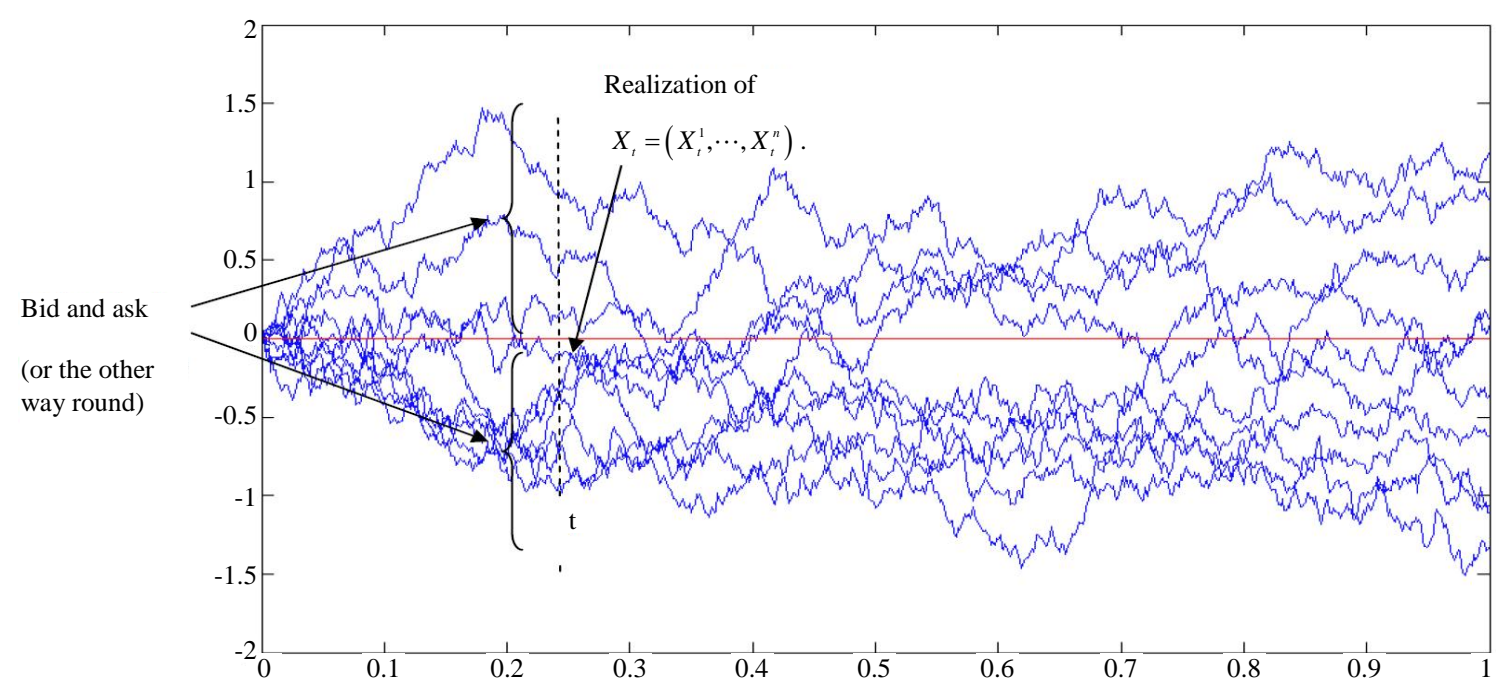

Figure 1. Realization of bid and ask.

$$
\operatorname{Ask}(t):=\max \{R(t),-T(t)\}
$$

Given Bid $(t)$ and $A s k(t)$, the market makers will agree on a stock price within the Bid/Ask spread, that is

$$
S(t)=\alpha(t) \operatorname{Bid}(t)+(1-\alpha(t)) \operatorname{Ask}(t)
$$

where $\alpha(t)$ is a stochastic process such that $0 \leq \alpha(t) \leq 1$. One could choose e.g., $\alpha(t)=\sigma(t)$ for a function $\sigma:[0, T] \rightarrow[0,1]$ or $\alpha(t)=f(R(t), T(t))$ for a function $f: \mathbb{R} \times \mathbb{R} \rightarrow[0,1]$.

For convenience, we will from now on assume that $\alpha(t) \equiv 1 / 2$, that is

$$
S_{t}=\frac{\operatorname{Bid}(t)+\operatorname{Ask}(t)}{2}=\frac{R(t)-T(t)}{2} .
$$

\section{Markovian Property of Processes $R, T$ and $S$}

For convenience, let us briefly discuss the Markovian property of the processes $\{R(t)\}_{0 \leq t \leq T}, \quad\{T(t)\}_{0 \leq t \leq T}$ and $\{S(t)\}_{0 \leq t \leq T}$ in some particular cases. The two cases considered here are the cases when the process $\left\{X_{i}(t)\right\}_{0 \leq t \leq T}$ are Brownian motions or Ornstein-Uhlenbeck processes. Let us first have on the definition of semimartingales rank processes.

Definition 3.1 Let $X_{1}, \cdots, X_{n}$ be continuous semimartingales. For $1 \leq k \leq n$, the $k$-th rank process of $X_{1}, \cdots, X_{n}$ is defined by

$$
X^{(k)}=\max _{i_{1}<\cdots<i_{k}} \min \left(X_{i_{1}}, \cdots, X_{i_{k}}\right)
$$

where $1 \leq i_{1}$ and $i_{k} \leq n$.

Note that, according to Definition 3.1, for $t \in \mathbb{R}_{+}$,

$$
\max _{1 \leq i \leq n} X_{i}(t)=X^{(1)}(t) \geq X^{(2)}(t) \geq \cdots \geq X^{(n)}(t)=\min _{1 \leq i \leq n} X_{i}(t)
$$

so that at any given time, the values of the rank processes represent the values of the original processes arranged in descending order (i.e. the (reverse) order statistics).

Using Definition 3.1, we get

$$
\begin{aligned}
& R(t):=X^{(n)+}(t), \\
& T(t):=X^{(1) *}(t) .
\end{aligned}
$$




\subsection{The Brownian Motion Case}

Here we assume that the processes $\left\{X_{i}(t)\right\}_{0 \leq t \leq T}, 1 \leq i \leq n$ are independent Brownian motions.

Proposition 3.2 The process $R$ possesses the Markov property with respect to the filtration

$$
\mathscr{F}_{t}:=\mathscr{T}_{t}^{B} \cap \sigma(R(t) ; 0 \leq t \leq T) .
$$

Proof. Let $B$ be a one-dimensional Brownian motion. We first prove that $B^{+}=\max (B, 0)$ is a Markov process. Define the process

$$
Y(t)=\left(\begin{array}{c}
|B(t)| \\
B(t)
\end{array}\right) \in \mathbb{R}^{2} .
$$

Then $\{Y(t)\}_{0 \leq t \leq T}$ is a two dimensional Feller process.

Let $g\left(x_{1}, x_{2}\right)=\frac{1}{2}\left(x_{1}+x_{2}\right)$. One observes that $g: \mathbb{R}^{2} \rightarrow \mathbb{R}$ is a continuous and open map. Thus is follows from [14] (Remark 1, p. 327) that $B^{+}(t)=Y^{+}(t)=g(Y(t))$ is a Feller process, too.

The latter argument also applies to the n-dimensional case, that is $\tilde{Y}:=\left(B_{1}^{+}(t), \cdots, B_{n}^{+}(t)\right)$ is a Feller process. Since

$$
\begin{aligned}
f: \mathbb{R}^{n} & \rightarrow \mathbb{R} \\
\left(x_{1}, \cdots, x_{n}\right) & \mapsto \min \left(x_{1}, \cdots, x_{n}\right)
\end{aligned}
$$

is a continuous and open map we conclude that $R(t)=f(\tilde{Y})$ is a Feller process.

Proposition 3.3 The process $T$ possesses Markov property with respect to the filtration

$$
\mathscr{T}_{t}:=\mathscr{F}_{t}^{B} \cap \sigma(T(t) ; 0 \leq t \leq T) .
$$

Proof. See the proof of Proposition 3.2.

Corollary 3.4 The process $S$ possesses Markov property with respect to the filtration

$$
\mathscr{T}_{t}:=\mathscr{F}_{t}^{B} \cap \sigma(S(t) ; 0 \leq t \leq T) .
$$

Proof. The process $Z$ defined by $Z_{t}=R_{t}+T_{t}$ for all $t \geq 0$ is a Markov process as sum of two Markov processes.

\subsection{The Ornstein-Uhlenbeck Case}

Here we assume that the process $X(t)=\left(X_{1}(t), \cdots, X_{n}(t)\right)$ is an n-dimensional Ornstein-Uhlenbeck, that is

$$
\mathrm{d} X_{i}(t)=-\alpha_{i} X_{i}(t) \mathrm{d} t+\sigma_{i} \mathrm{~d} B_{i}(t), 1 \leq i \leq n,
$$

where $\alpha_{i}$ and $\sigma_{i}$ are parameters. It is clear that an Ornstein-Uhlenbeck process is a Feller process. So we obtain

Proposition 3.5 The process $R, T$ and $S$ defined by (8) and (5) possess Markov property.

Proof. The conclusion follows from the proof of Proposition 3.2.

Remark 3.6 Using continuous and open transformations of Markov processes, the above results can be generalized to the case, when the bid and ask processes are Feller processes. See [14].

\section{Further Properties of $S(t)$}

In this Section, we want to use the semimartingale decomposition of our price process $S_{t}$ to analyze completeness and arbitrage on market driven by such a process.

We need the following result. See [15] (Proposition 4.1.11). See also [16] for the continuous semimartingales case and [17] for general semimartingales.

Theorem 4.1 Let $X_{1}, \cdots, X_{n}$ be continuous semimartingales of the form (1). For $k \in\{1,2, \cdots, n\}$, let $u(k)=\left(u_{t}(k), t \geq 0\right): \Omega \times[0, \infty[\rightarrow\{1,2, \cdots, n\}$ be any predictable process with the property: 


$$
X^{(k)}(t)=X_{u_{t}(k)}(t) .
$$

Then the $k$-th rank processes $X^{(k)}, k=1, \cdots, n$, are semimartingales and we have:

$$
\begin{aligned}
X^{(k)}(t)= & X^{(k)}(0)+\sum_{i=1}^{n} \int_{0}^{t} 1_{\left\{u_{s}(k)=i\right\}} \mathrm{d} X_{i}(s)+\frac{1}{2} \sum_{i=1}^{n} \int_{0}^{t} 1_{\left\{u_{s}(k)=i\right\}} \mathrm{d} L_{s}^{0}\left(\left(X^{(k)}-X_{i}\right)^{+}\right) \\
& -\frac{1}{2} \sum_{i=1}^{n} \int_{0}^{t} 1_{\left\{u_{s}(k)=i\right\}} \mathrm{d} L_{s}^{0}\left(\left(X^{(k)}-X_{i}\right)^{-}\right),
\end{aligned}
$$

where $L_{t}^{0}(X)$ is the local time of the semimartingale $X$ at zero, defined by

$$
\left|X_{t}\right|=\left|X_{0}\right|+\int_{0}^{t} \operatorname{sgn}\left(X_{s-}\right) \mathrm{d} X_{s}+L_{t}^{0}(X),
$$

where $\operatorname{sgn}(x)=-1_{(-\infty, 0]}(x)+1_{(0, \infty)}(x)$.

For completeness, we give the proof of the proposition.

Proof. We find that

$$
X_{t}^{(k)}-X_{0}^{(k)}=\sum_{i=1}^{n} \int_{0}^{t} 1_{\left\{u_{s}(k)=i\right\}} \mathrm{d} X_{s}^{i}+\sum_{i=1}^{n} \int_{0}^{t} 1_{\left\{u_{s}(k)=i\right\}} \mathrm{d}\left(X_{s}^{(k)}-X_{s}^{i}\right),
$$

where we used the property $\sum_{i=1}^{n} 1_{\left\{u_{s}(k)=i\right\}}=1$. It follows,

$$
\begin{aligned}
X_{t}^{(k)}-X_{0}^{(k)}= & \sum_{i=1}^{n} \int_{0}^{t} 1_{\left\{u_{s}(k)=i\right\}} \mathrm{d} X_{s}^{i}+\sum_{i=1}^{n} \int_{0}^{t} 1_{\left\{u_{s}(k)=i\right\}} \mathrm{d}\left(X_{s}^{(k)}-X_{s}^{i}\right)^{+} \\
& -\sum_{i=1}^{n} \int_{0}^{t} 1_{\left\{u_{s}(k)=i\right\}} \mathrm{d}\left(X_{s}^{(k)}-X_{s}^{i}\right)^{-} .
\end{aligned}
$$

We note the fact

$$
\left\{u_{s}(k)=i\right\} \subset\left\{X_{s}^{(k)}=X_{i}(s)\right\} .
$$

We now use the following formula:

$$
\frac{1}{2} L_{t}^{0}(X)=\int_{0}^{t} 1_{\left\{X_{s}=0\right\}} \mathrm{d} X_{s},
$$

which is valid for non-negative semimartingales $X$. See, e.g., $[15,18]$

Then, by applying (14) to $\left(X^{(k)}(t)-X_{i}(t)\right)^{ \pm}, t \geq 0,(12)$ becomes:

$$
\begin{aligned}
X^{(k)}(t)-X^{(k)}(0)= & \sum_{i=1}^{n} \int_{0}^{t} 1_{\left\{u_{s}(k)=i\right\}} \mathrm{d} X_{i}(s)+\frac{1}{2} \sum_{i=1}^{n} \int_{0}^{t} 1_{\left\{u_{s}(k)=i\right\}} \mathrm{d} L_{s}^{0}\left(\left(X^{(k)}-X_{i}\right)^{+}\right) \\
& -\frac{1}{2} \sum_{i=1}^{n} \int_{0}^{t} 1_{\left\{u_{s}(k)=i\right\}} \mathrm{d} L_{s}^{0}\left(\left(X^{(k)}-X_{i}\right)^{-}\right) .
\end{aligned}
$$

Then proof is completed.

\subsection{The Brownian Motion Case}

If $X_{i}(t)=B_{i}^{+}(t)$ or $B_{i}^{*}(t), i=1, \cdots, n$ are $n$ independent Brownian motions, the evolution of $R(t)$ and $T(t)$ follows from Theorem 4.1.

Corollary 4.2 Let the processes $\{R(t)\}_{t \geq 0}$ and $\{T(t)\}_{t \geq 0}$ be given by Equation (8). Then $R(t)=B^{(n)+}(t)$ and $T(t)=B^{(1) *}(t)$ and we have:

$$
\begin{aligned}
R(t) & =R(0)+\sum_{i=1}^{n} \int_{0}^{t} 1_{\left\{u_{s}(n)=i\right\}}\left\{\mathrm{d} B_{i}^{+}(s)-\frac{1}{2} \mathrm{~d} L_{s}^{0}\left(B_{i}^{+}-R\right)\right\} \\
& =R(0)+\sum_{i=1}^{n} \int_{0}^{t} 1_{\left\{u_{s}(n)=i\right\}}\left\{1_{\left\{B_{i}(s)>0\right\}} \mathrm{d} B_{i}(s)+\frac{1}{2}\left[\mathrm{~d} L_{s}^{0}\left(B_{i}\right)-\mathrm{d} L_{s}^{0}\left(B_{i}^{+}-R\right)\right]\right\},
\end{aligned}
$$


and

$$
\begin{aligned}
T(t) & =T(0)+\sum_{i=1}^{n} \int_{0}^{t} 1_{\left\{v_{s}(n)=i\right\}}\left\{\mathrm{d} B_{i}^{*}(s)+\frac{1}{2} \mathrm{~d} L_{s}^{0}\left(T-B_{i}^{*}\right)\right\} \\
& =T(0)+\sum_{i=1}^{n} \int_{0}^{t} 1_{\left\{v_{s}(n)=i\right\}}\left\{1_{\left\{B_{i}(s) \leq 0\right\}} \mathrm{d} B_{i}(s)+\frac{1}{2}\left[\mathrm{~d} L_{s}^{0}\left(T-B_{i}^{*}\right)-\mathrm{d} L_{s}^{0}\left(B_{i}\right)\right]\right\} .
\end{aligned}
$$

We can rewrite $R(t)$ and $T(t)$ as follows:

$$
\begin{aligned}
& R(t)=R(0)+M^{R}(t)+V^{R}(t), \\
& T(t)=T(0)+M^{T}(t)+V^{T}(t),
\end{aligned}
$$

where $M^{R}(t), M^{T}(t)$ are continuous local martingales and $V^{R}(t), V^{T}(t)$ are continuous processes of locally bounded variation given by:

$$
\begin{aligned}
V^{R}(t) & =\sum_{i=1}^{n} \int_{0}^{t} 1_{\left\{u_{s}(n)=i\right\}} \frac{1}{2}\left\{\mathrm{~d} L_{s}^{0}\left(B_{i}\right)-\mathrm{d} L_{s}^{0}\left(B_{i}^{+}-R\right)\right\}, \\
M^{R}(t) & =\sum_{i=1}^{n} \int_{0}^{t} 1_{\left\{u_{s}(n)=i\right\}} 1_{\left\{B_{i}(s)>0\right\}} \mathrm{d} B_{i}(s), \\
V^{T}(t) & =\sum_{i=1}^{n} \int_{0}^{t} 1_{\left\{v_{s}(1)=i\right\}} \frac{1}{2}\left\{\mathrm{~d} L_{s}^{0}\left(T-B_{i}^{*}\right)-\mathrm{d} L_{s}^{0}\left(B_{i}\right)\right\}, \\
M^{T}(t) & =\sum_{i=1}^{n} \int_{0}^{t} 1_{\left\{v_{s}(n)=i\right\}} 1_{\left\{B_{i}(s) \leq 0\right\}} \mathrm{d} B_{i}(s) .
\end{aligned}
$$

The following corollary gives the semimartingale decomposition satisfied by the process $S$.

Corollary 4.3 Assume that the process $S=\{S(t)\}_{0 \leq t \leq T}$ is given by (5). Then one can write $S(t)=f(A(t))$ where $A(t)=(R(t), T(t))$ and $f\left(x_{1}, x_{2}\right)=\frac{1}{2}\left(x_{1}-x_{2}\right)$, and we have:

$$
\begin{aligned}
S(t)= & S(0)+\frac{1}{2} \sum_{i=1}^{n} \int_{0}^{t}\left(1_{\left\{u_{s}(n)=i\right\}} 1_{\left\{B_{i}(s)>0\right\}}-1_{\left\{v_{s}(n)=i\right\}} 1_{\left\{B_{i}(s) \leq 0\right\}}\right) \mathrm{d} B_{i}(s) \\
& +\frac{1}{2} \sum_{i=1}^{n} \int_{0}^{t}\left(1_{\left\{u_{s}(n)=i\right\}}+1_{\left\{v_{s}(n)=i\right\}}\right) \mathrm{d} L_{s}^{0}\left(B_{i}\right) \\
& -\frac{1}{2} \sum_{i=1}^{n}\left\{\int_{0}^{t} 1_{\left\{u_{s}(n)=i\right\}} \mathrm{d} L_{s}^{0}\left(B_{i}^{+}-R\right)+\int_{0}^{t} 1_{\left\{v_{s}(n)=i\right\}} \mathrm{d} L_{s}^{0}\left(T-B_{i}^{*}\right)\right\} .
\end{aligned}
$$

In order to price options with respect to $S(t)$, one should ensure that $S(t)$ does not admit arbitrage possibilities and the natural question which arises at this point is the following: Can we find an equivalent probability measure $Q$ such that, $S$ is a $Q$-sigma martingale? Since the process $S$ is continuous, we can reformulate the question as: Can we find an equivalent probability measure $Q$ such that, $S$ is a $Q$ local martingale ${ }^{1}$ ?

We first give the following useful remark; See [19] (Theorem 1).

Remark 4.4 Let $X(t)=X_{0}+M(t)+V(t)$ be a continuous semimartingale on a filtered probability space $\left(\Omega, \mathscr{F},\left\{\mathscr{T}_{t}\right\}_{0 \leq t \leq T}, P\right)$. Let $C_{t}=[X, X]_{t}=[M, M]_{t}, 0 \leq t \leq T$. A necessary condition for the existence of an equivalent martingale measure is that $\mathrm{d} V \ll \mathrm{d} C$.

Consequence 4.5 Since local time is singular, we observe that the total variation of the bounded variation part in 21 cannot be absolutely continuous with respect to the quadratic variation of the martingale. It follows that, the set of equivalent martingale measures is empty, and thus, such a market contains arbitrage opportunities.

${ }^{1}$ In fact since $S$ is continuous and since all continuous sigma martingales are in fact local martingales, we only need to concern ourselves with local martingales 


\section{2. (In)complete Market with Hidden Arbitrage}

In this Section, we consider a model with $\{S(t)\}_{t>0}$, denoting a stochastic process modeling the price of a risky asset, and $\{R(t)\}_{0 \leq t \leq T}$ denotes the value of a risk free money market account. We assume a given filtered probability space $\left(\Omega, \mathscr{T},\left\{\mathscr{T}_{t}\right\}_{0 \leq t \leq T}, P\right)$, where $\left\{\mathscr{F}_{t}\right\}_{0 \leq t \leq T}$ satisfies the "usual hypothesis". In such a market, a trading strategy $(a, b)$ is self-financing if $a$ is predictable, $b$ is optional, and

$$
a(t) S(t)+b(t) R(t)=a(0) S(0)+b(0) R(0)+\int_{0}^{t} a(s) \mathrm{d} S(s)+\int_{0}^{t} b(s) \mathrm{d} R(s)
$$

for all $0 \leq t \leq T$. For simplicity, we let $S_{0}=0$ and $R(t) \equiv 1$ (thus the interest rate $r=0$ ), so that $\mathrm{d} R(t)=0$, and (22) becomes

$$
a(t) S(t)+b(t) R(t)=b(0)+\int_{0}^{t} a(s) \mathrm{d} S(s)
$$

Definition 4.6 (See [13])

- We call a random variable $H \in \mathscr{T}_{T}$ a contingent claim. Further, a contingent claim $H$ is said to be $Q$ -redundant if, for a probability measure $Q$, there exists a self-financing strategy $(a, b)$ such that

$$
V^{Q}(t)=E_{Q}\left[H \mid \cdot \mathscr{T}_{t}\right]=b(0)+\int_{0}^{t} a(s) \mathrm{d} S(s),
$$

where $\{V(t)\}_{0 \leq t \leq T}$ is the value of the portfolio.

- A market $(S(t), R(t))=(S(t), 1)$ is $Q$-complete if every $H \in L^{1}\left(\mathscr{F}_{T}, Q\right)$ is $Q$-redundant.

Define the process $\left(M^{S}(t)\right)_{t>0}$ as follows

$$
M^{S}(t)=\frac{1}{2} \sum_{i=1}^{n} \int_{0}^{t}\left(1_{\left\{u_{s}(n)=i\right\}} 1_{\left\{B_{i}(s)>0\right\}}-1_{\left\{v_{s}(n)=i\right\}} 1_{\left\{B_{i}(s) \leq 0\right\}}\right) \mathrm{d} B_{i}(s) .
$$

Then the following theorem is immediate from [13] (Theorem 3.2).

Theorem 4.7 Suppose that there exists a unique probability measure $P^{*}$ equivalent to $P$ such that $M^{S}(t)$ is a $P^{*}$-local martingale. Then, the market $(S(t), 1)$ is $P^{*}$-complete.

Proof. Omitted.

Proposition 4.8 Suppose that $n \geq 2$. Then, there exists no unique martingale measure $P^{*}$ such that $M^{S}(t)$ is a $P^{*}$-local martingale.

Proof. From (24), we observe that $M^{S}(t)$ is a $P$-martingale. Let us construct another equivalent martingale measure $P^{*}$. For this purpose, assume without loss of generality that $u_{s}(n)$ and $v_{s}(n)$ are given by

$$
u_{s}(n)=\min \left\{i \in\{1, \cdots, n\}: B_{i}^{+}(t)=R(t)\right\}
$$

and

$$
v_{s}(n)=\min \left\{i \in\{1, \cdots, n\}: B_{i}^{*}(t)=T(t)\right\} .
$$

Now define the process $h$ as

$$
h(t)=1_{\{A(t)\}},
$$

where

$$
A(t)=\{\omega \in \Omega: \beta(t, \omega)=0\}
$$

with

$$
\beta(s)=\sum_{i=1}^{n}\left(1_{\left\{u_{s}(n)=i\right\}} 1_{\left\{B_{i}(s)>0\right\}}-1_{\left\{v_{s}(n)=i\right\}} 1_{\left\{B_{i}(s) \leq 0\right\}}\right) .
$$

One finds that $\operatorname{Pr}[A(t)]>0$ for all $t$. Let us define the equivalent measure $P^{*}$ with respect to a density 
process $Z_{t}$ given by

$$
Z_{t}=\mathscr{E}[N]_{t}
$$

Here, $\mathscr{E}(N)$ denotes the Doléans-Dade exponential of the martingale $N_{t}$ defined by

$$
N_{t}=\sum_{i=1}^{n} \int_{0}^{t} h(s) \mathrm{d} B_{i}(s)
$$

Then, it follows from the Girsanov-Meyer theorem (see [20]) that $M^{S}(t)$ has a $P^{*}$-semimartingale decomposition with a bounded variation part given by $\int_{0}^{t} 2 h(s) \mathrm{d}\left\langle M^{s}, M^{s}\right\rangle_{s}$. We have that

$$
\int_{0}^{t} 2 h(s) \mathrm{d}\left\langle M^{s}, M^{s}\right\rangle_{s}=\frac{1}{2} \int_{0}^{t} h(s) \beta(s) \mathrm{d} s .
$$

Since $h \beta=0$, it follows that

$$
\int_{0}^{t} h(s) \mathrm{d}\left\langle M^{S}, M^{S}\right\rangle_{s}=0
$$

Thus $M^{S}(t)$ is a $P^{*}$-martingale. Since $P$ is also a martingale measure with $P \neq P^{*}$, the result follows.

Remark 4.9 In the case $n=1$ (a single Bid/Ask), the market becomes complete since the process $\beta(t)$, defined by Equation (25) in the proof is equal to $\operatorname{sgn}(B(t))$. Therefore the unique martingale measure is $P$.

We can then deduce the following theorem on our process $S(t)$.

Then

Theorem 4.10 Suppose that $S=\{S(t)\}_{0 \leq t \leq T}$ and $\left\{M^{S}(t)\right\}_{0 \leq t \leq T}$ are given by (21) and (24), respectively.

- For $n=1$ (a single Bid/Ask), the market $(S(t), 1)$ is $P$-complete and admits the arbitrage opportunity (26).

- For $n \geq 2$ (more than a single Bid/Ask), the market $(S(t), 1)$ is incomplete and arbitrage exists.

Proof. From Theorem 4.8, we know that the market is $P$-complete for $n=1$ and incomplete for $n>1$. Let

$P$ such that $M^{S}(t)$ is a $P$-local martingale. For $n=1$, let us construct an arbitrage strategy. Let

$$
a_{s}=1_{\left\{\operatorname{supp}\left(\mathrm{d}\left[M^{S}, M^{s}\right]\right)\right\}^{c}}(s),
$$

where $\operatorname{supp}\left(\mathrm{d}\left[M^{S}, M^{S}\right]\right)$ denotes the $\omega$ by $\omega$ support of the (random) measure $\mathrm{d}\left[M^{S}, M^{S}\right]_{s}(\omega)$; that is, for fixed $\omega$ it is the smallest closed set in $\mathbb{R}_{+}$such that $\mathrm{d}\left[M^{s}, M^{s}\right]_{s}$ does not charge its complement. (Compare with the proof of Proposition 4.8.) Let

$$
\begin{aligned}
H=H(T)= & \frac{1}{2} \sum_{i=1}^{n} \int_{0}^{T}\left(1_{\left\{u_{s}(n)=i\right\}}+1_{\left\{v_{s}(n)=i\right\}}\right) \mathrm{d} L_{s}^{0}\left(B_{i}\right) \\
& -\frac{1}{2} \sum_{i=1}^{n}\left\{\int_{0}^{T} 1_{\left\{u_{s}(n)=i\right\}} \mathrm{d} L_{s}^{0}\left(B_{i}^{+}-R\right)+\int_{0}^{T} 1_{\left\{v_{s}(n)=i\right\}} \mathrm{d} L_{s}^{0}\left(T-B_{i}^{*}\right)\right\} .
\end{aligned}
$$

Assume without loss of generality that $H \in L^{1}\left(\mathscr{F}_{T}, P\right)$. Then, by Theorem 4.7, there exists a self financing strategy $\left(j_{t}, b\right)$ such that $H=H(T)=E[H(T)]+\int_{0}^{T} j(s) \mathrm{d} S(s)$. However, from (26), we also have $H_{T}=0+\int_{0}^{T} a(s) \mathrm{d} H(s)$. Moreover, we have $\int_{0}^{t} a(s) \mathrm{d} M^{s}(s)=0,0 \leq t \leq T$, by construction of the process $a$. Hence, $H=H(T)=0+\int_{0}^{T} a(s) \mathrm{d} S(s)$, which is an arbitrage opportunity.

Remark 4.11 We do not make the assumption that we are working in an arbitrage free market, rather, we define the notion of redundancy (see [13] Definition 2.1), which in some sense is equivalent to the notion of replication with the difference that, replication is on a arbitrage free setting.

\section{Pricing and Insider Trading with Respect to $S(t)$}

In this Section, we discuss a framework introduced in [21], which enables us pricing of contingent claims with respect to the price process $S(t)$ of the previous sections. We even consider the case of insider trading, that is, 
the case of an investor, who has access to insider information. To this end, we need some notions.

We consider a market driven by the stock price process $S(t)$ on a filtered probability space

$\left(\Omega, \mathscr{C},\left\{\mathscr{H}_{t}\right\}_{0 \leq t \leq T}, \mathbb{P}\right)$. We assume that, the decisions of the trader are based on market information given by the filtration $\mathscr{G}=\left\{\mathscr{T}_{t}\right\}_{0 \leq t \leq T}$ with $\mathscr{H}_{t} \subset \mathscr{C}_{t}$ for all $t \in[0, T], T>0$ being a fixed terminal time. In this context an insider strategy is represented by an $\mathscr{G}$-adapted process $\{\varphi(t)\}_{0 \leq t \leq T}$ and we interpret all anticipating integrals as the forward integral; See, for e.g., [22,23] for more details. In such a market, a natural tool to describe the self-financing portfolio is the forward integral of an integrand process $Y$ with respect to an integrator $S$, denoted by $\int_{0}^{t} Y \mathrm{~d}^{-} S$; See [23]. The following definitions and concepts are consistent with those given in [21].

Definition 5.1 A self-financing portfolio is a pair $\left(V_{0}, a\right)$, where $V_{0}$ is the initial value of the portfolio and

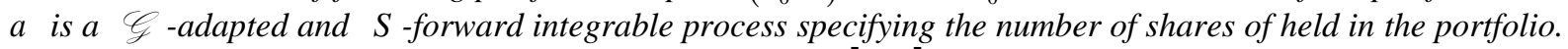
The market value process $V$ of such a portfolio at time $t \in[0, T]$, is given by

$$
V(t)=V_{0}+\int_{0}^{t} a(s) \mathrm{d}^{-} S(s)
$$

while $b(t)=V(t)-S(t) a(t)$ constitutes the number of shares of the less risky asset held.

\section{1. $\mathscr{A}$-Martingales}

Now, we briefly review the definition of $/ /$-martingales which generalizes the concept of a martingale. We refer to [21] for more information about this notion. Throughout this Section, $\mathscr{A}$ will be a real linear space of measurable processes indexed by $[0,1)$ with paths which are bounded on each compact interval of $[0,1)$.

Definition 5.2 A process $X=\{X(t)\}_{0 \leq t \leq T}$ is said to be a./ -martingale, if every $\theta$ in $/$ is $X$ -improperly forward integrable and

$$
E\left[\int_{0}^{t} \theta(s) \mathrm{d}^{-} X(s)\right]=0 \text { for every } 0 \leq t \leq T .
$$

Definition 5.3 A process $X=\{X(t)\}_{0 \leq t \leq T}$ is said to be $/$-semimartingale if it can be written as the sum of an $\mathscr{C}$-martingale $M$ and a bounded variation process $V$, with $V(0)=0$.

\section{Remark 5.4}

- Let $X$ be a continuous $\mathscr{C}$-martingale with $X$ belonging to $\mathscr{C}$, then, the quadratic variation of $X$ exists improperly. In fact, if $\int_{0} X(t) \mathrm{d}^{-} X(t)$ exists improperly, then one can show that $[X, X]$ exists improperly and $[X, X]=X^{2}-X^{2}(0)-2 \int_{0}^{1} X(s) \mathrm{d}^{-} X(s)$.

- Let $X$ a continuous square integrable martingale with respect to some filtration $\mathscr{F}$. Suppose that every process in $\mathscr{A}$ is the restriction to $[0, T)$ of a process $(\theta(t), 0 \leq t \leq T)$ which is $\mathscr{T}$-adapted. Moreover, suppose that its paths are left continuous with right limits and $E\left[\int_{0}^{T} \theta^{2}(t) \mathrm{d}[X]_{t}\right]<\infty$. Then $X$ is an
$\mathscr{C}$-martingale.

\subsection{Completeness and Arbitrage: $\mathscr{A}$-Martingale Measures}

The subsequent definitions and notions are from [21].

Definition 5.5 Let $h$ be a self-financing portfolio in . $/$, which is $S$-improperly forward integrable and $X$ its wealth process. Then $h$ is an $\mathscr{C}$-arbitrage if $X(T)=\lim _{t \rightarrow T} X(t)$ exists almost surely, $\operatorname{Pr}[X(T) \geq 0]=1$ and $\operatorname{Pr}[X(T)>0]>0$.

Definition 5.6 If there is no $/$-arbitrage, the market is said to be $/$-arbitrage free.

Definition 5.7 A probability measure $Q \sim P$ is called $a . /$-martingale measure if with respect to $Q$ the process $S$ is an $\mathscr{A}$-martingale according to Definition 5.2.

We need need the following assumption. See [21].

Assumption 5.8 Suppose that for all $h$ in $\mathscr{A}$ the following condition holds. Then $h$ is $S$-improperly forward integrable and

$$
\int_{0}^{1} \mathrm{~d}^{-} \int_{0}^{t} h(s) \mathrm{d}^{-} S(s)=\int_{0}^{h} h(t) \mathrm{d}^{-} S(t)=\int_{0}^{h} h(t) \mathrm{d}^{-} \int_{0}^{t} \mathrm{~d}^{-} S(s)
$$

The proof of the following proposition can be found in [21]. 
Proposition 5.9 Under Assumption 5.8, if there exists an ./ -martingale measure $Q$, the market is -arbitrage free.

Definition 5.10 A contingent claim is an $\mathscr{T}$-measurable random variable. Let $\mathscr{L}$ be the set of all contingent claims the investor is interested in.

Definition 5.11

- A contingent claim $C$ is called $\mathscr{C}$-attainable if there exists a self-financing trading portfolio $(X(0), h)$ with $h$ in $\mathscr{C}$, which is $S$-improperly forward integrable, and whose terminal portfolio value coincides with $C$, i.e., $\lim _{t \rightarrow T} X(t)=C$ P-a.s.

Such a portfolio strategy $h$ is called a replicating or hedging portfolio for $C$, and $X(0)$ is the replication price for $C$.

- A $\mathscr{C}$-arbitrage free market is called $(\mathscr{C}, \mathscr{L})$-complete if every contingent claim in $L$ is attainable.

Assumption 5.12 For every $\mathscr{C}_{0}$-measurable random variable $\eta$, and $h$ in $\mathscr{A}$ the process $u=h \eta$, belongs to . .

Proposition 5.13 Suppose that the market is $/$-arbitrage free, and that Assumption 5.8 holds. Then the replication price of an attainable contingent claim is unique.

Proof. Let $Q$ be a given measure equivalent to $P$. For such a $Q$, let $\mathscr{A}$ be a set of all strategies ( $/ C_{t}-$ adapted) such that (28) in Definition 5.2 is satisfied. Then, it follows from Proposition 5.9 that the market $(S(t), 1)$ in Section 4.2 is $\mathscr{C}$-arbitrage free.

Next, we shall discuss attainability of claims in connection with a concrete set $\mathscr{C}$ of trading strategies.

\subsection{Hedging with Respect to $S(t)$}

In this Section, we want to determine hedging strategies for a certain class of European options with respect to the price process $S(t)$ of Section 4.2. Let us now assume that $n=1$ (a single Bid/Ask). Then, the price process $S$ is the sum of a Wiener process and a continuous process with zero quadratic variation; moreover, we have that $\mathrm{d}[S]_{t}=\frac{1}{4} \beta^{2}(t)=\frac{1}{4}$, where $\beta(t)$ is given by (25). We can derive the following proposition which is similar to [21] (Proposition 5.29).

Proposition 5.14 Let $\psi$ be a function in $C^{0}(\mathbb{R})$ of polynomial growth. Suppose that there exist $(v(t, x), 0 \leq t \leq T, x \in \mathbb{R})$ of class $C^{1,2}([0, T) \times \mathbb{R}) \cap C^{0}([0, T] \times \mathbb{R})$ which is a solution of the following Cauchy problem

$$
\left\{\begin{array}{l}
\partial_{t} v(t, x)+\frac{1}{8} \partial_{y y} v(t, y)=0 \text { on }[0, T) \times \mathbb{R}, \\
v(T, y)=\psi(y) .
\end{array}\right.
$$

Set

$$
h(t)=\partial_{y} v(t, S(t)), 0 \leq t \leq T, X(0)=v(0, S(0)) .
$$

Then $(X(0) ; h)$ is a self-financing portfolio replicating the contingent claim $(\psi S(T))$.

In particular, $(S(t), 1)$ is $\mathscr{C}, \mathscr{L}$-complete, where $\mathscr{C}$ is given by

$$
\begin{aligned}
\mathscr{C}=\{( & (t, S(t)), 0 \leq t \leq T): \phi:[0, T] \times \mathbb{R} \rightarrow \mathbb{R}, \text { Borel measurable, } \\
& \text { of polynomial growth and lower bounded }\}
\end{aligned}
$$

and $\mathscr{L}$ by all claims as stated in this Proposition.

\section{Conclusion}

Proof. The proof is a direct consequence of Itô's Lemma for forward integrals. See [21] (Proposition 5.29).

In this paper, assuming that the dynamics of the bid and ask prices are given by Itô processes, we derive the stochastic differential equation satisfied by the "best bid" and the "best ask" from which we get the dynamic of the middle (stock) price. The evolution of the latter is given by a semimartingale, whose final variation part, is not absolute continuous with respect to the Lebesgue measure. We then show that, such a market admits a 
hidden arbitrage opportunity and compute the arbitrage strategy. We also discuss the notion of (insider) hedging in this market.

\section{REFERENCES}

[1] J. Harrison and D. Kreps, “Martingales and Arbitrage in Multiperiod Securities Markets,” Journal of Economic Theory, Vol. 20, No. 3, 1979, pp. 381-408. http://dx.doi.org/10.1016/0022-0531(79)90043-7

[2] J. Harrison and S. Pliska, "Martingales and Stochastic Integrals in the Theory of Continuous Trading," Stochastic Processes and Their Applications, Vol. 11, No. 3, 1979, pp. 215-260. http://dx.doi.org/10.1016/0304-4149(81)90026-0

[3] D. M. Kreps, “Arbitrage and Equilibrium in Economics with Infinitely Many Commmodities,” Journal of Mathematical Economics, Vol. 8, No. 1, 1981, pp. 15-35. http://dx.doi.org/10.1016/0304-4068(81)90010-0

[4] J. Bion-Nadal, "Bid-Ask Dynamic Pricing in Financial Markets with Transaction Costs and Liquidity Risk,” Journal of Mathematical Economics, Vol. 45, No. 11, 2009, pp. 738-750. http://dx.doi.org/10.1016/j.jmateco.2009.05.004

[5] E. Jouini and H. Kallal, "Martingales and Arbitrage in Security Market with Transaction Coast," Journal of Economic Theory, Vol. 66, No. 1, 1995, pp. 178-197. http://dx.doi.org/10.1006/jeth.1995.1037

[6] A. Cherny, “General Arbitrage Pricing Model. II. Transaction Costs,” In C. Donati-Martin, M. Emery, A. Rouault and C. Stricker, Eds., Séminaire de Probabilités XL, Volume 1899 of Lecture Note in Mathematics, Springer, Berlin, Heidelberg, 2007, pp. 447-461.

[7] P. Carr, H. Geman and D. Madan, “Pricing and Hedging in Incomplete Markets,” Journal of Financial Economics, Vol. 62, No. 1, 2001, pp. 131-167. http://dx.doi.org/10.1016/S0304-405X(01)00075-7

[8] H. Föllmer and A. Schield, "Stochastic Finance: An Introduction in Discrete Time,” 2nd Edition, De Gruyter Studies in Mathematics, Berlin, New York, 2004.

[9] N. El Karoui and M. C. Quenez, "Dynamic Programming and the Pricing of Contingent Claims in Incomplete Markets,” SIAM Journal on Control and Optimization, Vol. 33, No. 1, 1995, pp. 29-66. http://dx.doi.org/10.1137/S0363012992232579

[10] N. El Karoui, S. Peng and M. C. Quenez, “Backward Stochastic Differential Equations in Finance,” Mathematical Finance, Vol. 7, No. 1, 1997, pp. 1-77. http://dx.doi.org/10.1111/1467-9965.00022

[11] S. Peng, "Non Linear Expectation, Nonlinear Evaluation and Risk Measure, Volume 1856 of Lecture Notes in Mathematics,” Springer, Berlin, Heidelberg, 2004, pp.165-253.

[12] S. Peng, "Modelling Derivatives Pricing Mechanisms with Their Generating Functions,” 2006. http://arxiv.org/pdf/math/0605599v1.pdf

[13] R. Jarrow and P. Protter, “Large Traders, Hidden Arbitrage, and Complete Markets,” Journal of Banking \& Finance, Vol. 29, No. 11, 2005, pp. 2803-2820. http://dx.doi.org/10.1016/j.jbankfin.2005.02.005

[14] E. B. Dynkin, “Markov Processes, Volume 1,” Springer-Verlag, Berlin, Göttigen, Heidelberg, 1965.

[15] R. Fernholz, “Stochastic Portfolio Theory,” Springer-Verlag, Berlin, 2002. http://dx.doi.org/10.1007/978-1-4757-3699-1

[16] A. D. Banner and R. Ghomrasni, "Local Times of Ranked Continuous Semimartingales,” Stochastic Processes and their Applications, Vol. 118, No. 7, 2008, pp. 1244-1253. http://dx.doi.org/10.1016/j.spa.2007.08.001

[17] R.Ghomrasni and O. Menoukeu-Pamen, "Decomposition of Order Statistics of Semimartingales Using Local Times,” Stochastic Analysis and Applications, Vol. 28, No. 3, 2010, pp. 467-479. http://dx.doi.org/10.1080/07362991003708341

[18] R. J. Chitashvili and M. G. Mania, “Decomposition of the Maximum of Semimartingales and Generalized Itô's Formula,” In: V. V. Sazonov and T. L. Shervashidze, Eds., New Trends in Probability and Statistics, Volume 1, Proceedings of the Bakuriani Colloquium in Honour of Yu. V. Prohorov, USSR, Bakuriani, 24 February-4 March 1990, pp. 301-350.

[19] P. Protter and K. Shimbo, "No Arbitrage and General Semimartingales,” In: Markov Processes and Related Topics: A Festschrift for Thomas G. Kurtz, Volume 4, Institute of Mathematical Statistics, Beachwood, 2008, pp. 267-283.

[20] P. Protter, “Stochastic Integration and Differential Equations,” 2nd Edition, Springer-Verlag, Berlin, 2005. http://dx.doi.org/10.1007/978-3-662-10061-5

[21] R. Coviello and F. Russo, “Modeling Financial Assets without Semimartingale,” 2006. http://arxiv.org/pdf/math/0606642.pdf

[22] D. Nualart and E. Pardoux, "Stochastic Calculus with Anticipating Integrands," Probability Theory and Related Fields, Vol. 78, 1988, pp. 535-581. http://dx.doi.org/10.1007/BF00353876

[23] F. Russo and P. Vallois, “Forward, Backward and Symmetric Stochastic Integration,” Probability Theory and Related Fields, Vol. 97, No. 3, 1993, pp. 403-421. http://dx.doi.org/10.1007/BF01195073 